\title{
Dorsal root ganglion - a potential new therapeutic target for neuropathic pain
}

This article was published in the following Dove Press journal:

Journal of Pain Research

15 February 2012

Number of times this article has been viewed

\section{Damir Sapunar \\ Sandra Kostic \\ Adriana Banozic \\ Livia Puljak}

Department of Anatomy, Histology, and Embryology, University of Split Medical School, Soltanska 2, 21000 Split, Croatia
Correspondence: Damir Sapunar Department of Anatomy, Histology, and Embryology, University of Split Medical School, Soltanska 2, 21000 Split, Croatia Tel 38521557809 Fax 385 21 55781 I Email ds@mefst.hr
Abstract: A regional approach can protect our patients from often unacceptable adverse effects produced by systematically applied drugs. Regional therapeutic approaches, as well as interventions at the level of the peripheral nervous system and particularly the dorsal root ganglion (DRG), represent an alternative to the systemic application of therapeutic agents. This article provides an overview of DRG anatomical peculiarities, explains why the DRG is an important therapeutic target, and how animal models of targeted drug delivery can help us in the translation of basic research into clinical practice.

Keywords: dorsal root ganglion, neuropathic pain, pain therapy, targeted drug delivery

\section{Introduction}

The best available evidence indicates a major gap between an increasing understanding of the pathophysiology of pain and the disappointing inadequacy of its treatment. ${ }^{1}$ Unfortunately, physicians still rely on relatively ineffective analgesic drugs burdened with serious side effects. Meta-analyses of randomized clinical trials (RCTs) showed that only a few drugs are effective across the range of neuropathic pain disorders. ${ }^{2}$ Moreover, a limited number of basic research discoveries have been translated from animal pain models into effective pain therapy. ${ }^{3,4}$ Therefore, the translation of basic scientific discoveries into practical, clinical applications is a priority, as well as an improvement of treatment protocols and adjunctive therapies that promote greater effectiveness, patient adherence, or patient tolerance.

We are all well aware of the often unacceptable adverse effects produced by certain drugs when applied systematically or through the central nervous system (CNS). The regional therapeutic approach and interventions at the level of the peripheral nervous system, and particularly the dorsal root ganglion (DRG), represent an alternative to the systemic application of therapeutic agents. Many diseases of the peripheral nervous system are local rather than systemic (eg, trauma, cancer, zoster, radiculopathy) so the therapeutic approach can also be regional. In this review we will discuss what is already known about DRG targeted drug delivery in animal models and why the DRG is an important therapeutic target. The aim of this review is to foster the translation of basic research into clinical practice.

\section{Why the DRG is so special?}

The DRG is located between the dorsal root and the spinal nerve. It contains pseudounipolar neurons that convey sensory information from the periphery to 
the CNS. Following nerve injury or inflammation, these neurons may become an important source of increased nociceptive signaling through increased neuronal excitability and generation of ectopic discharges. ${ }^{5,6}$ This provides an excellent opportunity for the anesthesia of DRG neurons in order to prevent the development of these pathological discharges.

The DRG also lacks a protective surrounding capsular membrane, unlike the perineurium that protects the peripheral nerve fascicles and regulates their internal milieu. ${ }^{7}$ It has a permeable connective tissue capsule, which makes it an excellent choice for drug application. ${ }^{7}$ This permeability can be partly explained by a very high density of blood capillaries in the ganglion tissue. ${ }^{8}$ This rich blood supply may also explain why the DRG is tolerant to the intraneuronal local anesthetic injection, since toxic concentration cannot be easily reached due to high perfusion rates. ${ }^{9}$

As opposed to the blood-brain barrier in the CNS, the DRG and peripheral axons lack an efficient neurovascular barrier, which allows the easy diffusion of large molecular weight compounds in the interstitium surrounding the DRG neurons. $^{7}$ This absence of a vascular barrier exposes DRG neurons to toxic circulating agents, like intravenously applied antineoplastic drugs and antiretroviral agents, which thereby produce treatment-limiting peripheral neuropathy that is selective for sensory neurons in the DRG. ${ }^{10}$ The special position of the DRG also exposes them to injury. A close juxtaposition of sensitive neuronal and sturdy bone structures is probably the main reason for DRG injury (Figures 1 and 2). Arthritis of the zygapophyseal (facet) joint and the herniation of the intervertebral disc are the most common causes of radiculopathy. ${ }^{11}$ The chronic compression of the DRG or nearby nerve roots after vertebral injuries, intervertebral disc herniation, or intervertebral foramen stenosis is an important factor causing lower back pain and sciatica. In 1998 a method of producing chronic compression of the L5 DRG in rats was described, with expectations that this animal model will increase our understanding of lower back pain and sciatica. ${ }^{12}$ Peripheral or nerve root injury due to bone injury induces the synthesis and the release of numerous inflammatory mediators that can sensitize nociceptors and enhance pain. ${ }^{13,14}$

\section{Initial pathological changes in the DRG are the main triggers for neuropathic pain}

The most important changes responsible for the induction of neuropathic pain are found in the altered gene/protein

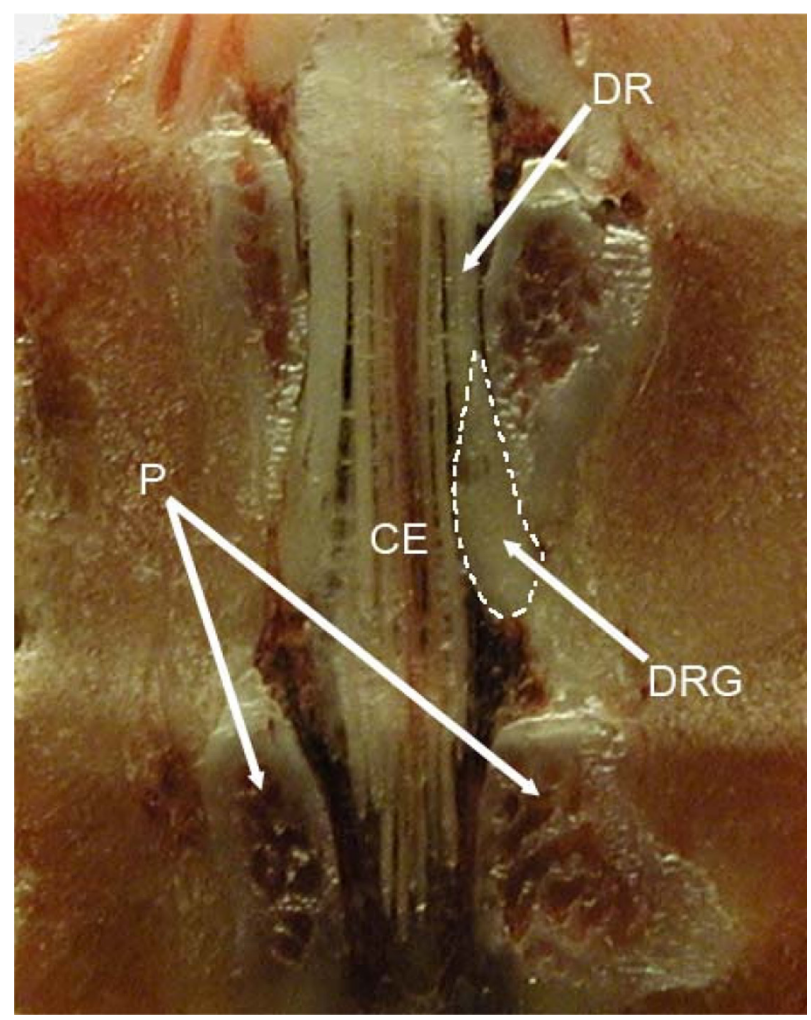

Figure I The position of the rat L5 DRG and its relation with surrounding bone structures and cauda equina.

Abbreviations: DRG, dorsal root ganglion; DR, dorsal root; $C E$, cauda equina; P, pedicles.

expression in primary sensory neurons. After damage to peripheral sensory fibers, upregulated expression of the $\mathrm{Ca}_{\mathrm{v}} \alpha_{2} \delta-_{1}$ channel subunit, the $\mathrm{Na}_{\mathrm{v}} 1.3$ sodium channel, and bradykinin (BK) B1 and capsaicin TRPV1 receptors in myelinated neurons contribute to hyperalgesia; while the downregulation of the $\mathrm{Na}_{\mathrm{v}} 1.8$ sodium channel, $\mathrm{B} 2$ receptor, substance P (SP), and even $\mu$-opioid receptors in unmyelinated neurons is responsible for the phenotypic switch in pain transmission. ${ }^{15}$

Many studies have focused on finding the source of abnormal spontaneous activity within DRG neurons. Voltage-gated sodium channels, which are essential for the generation and conduction of action potentials, are potential targets for treating neuropathic pain. Changes in their density, distribution, and functional activities following nerve injury were extensively studied. However, preclinical studies have shown unexpected results because most pain-associated voltagegated channels in the DRG are downregulated after peripheral nerve injury. The possible role of the DRG voltage-gated sodium channel function in neuropathic pain remains to be further investigated. ${ }^{16}$

Neurons are not the only players that drive the establishment and maintenance of common clinical pain states. 

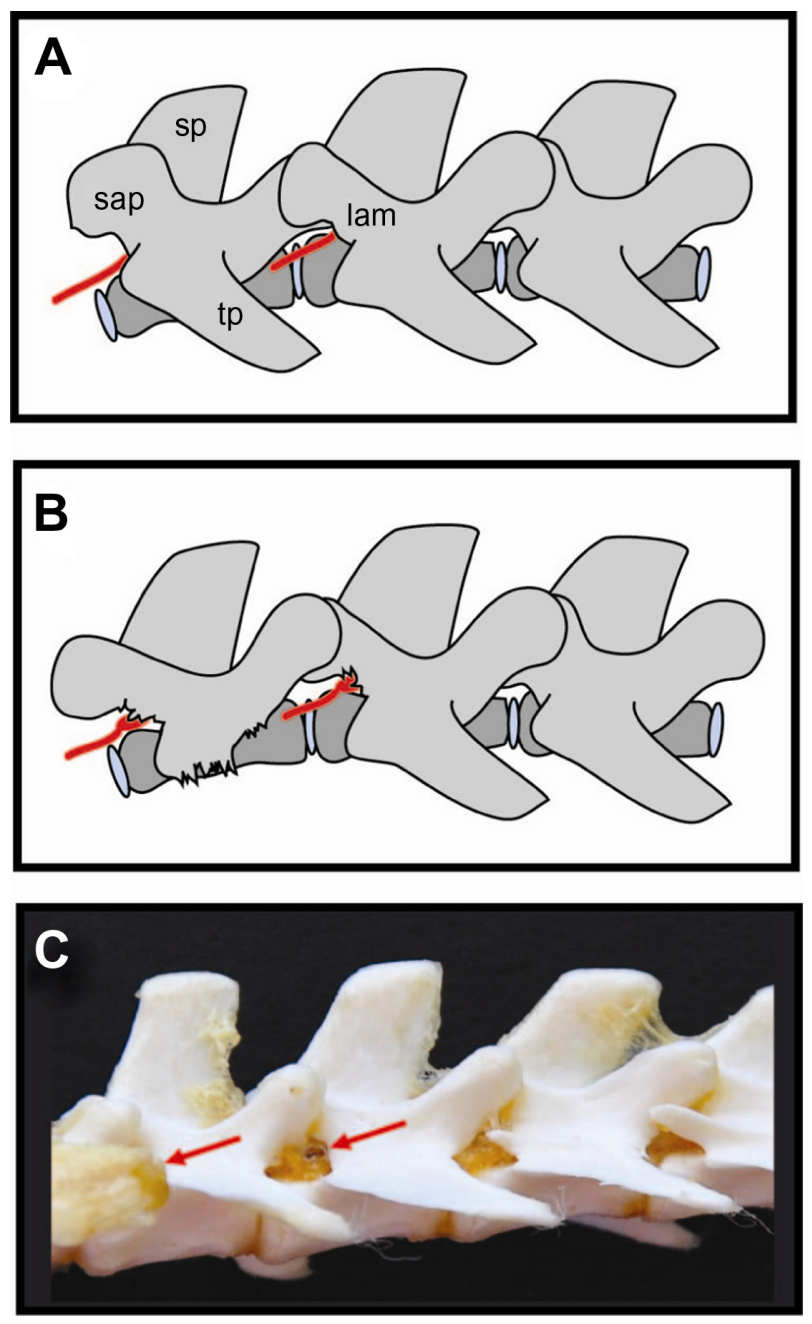

Figure 2 Schematic drawings (A and $\mathbf{B}$ ) and photo (C) of rat spine depicting position of dorsal root ganglia and surgical approach used for DRG injection. ${ }^{36}$ Reliably precise injection can be performed using minimal laminectomy, while blind needle-insertion approach requires a lot of skill and experience. The dorsal root ganglion is covered by laminar bone. (B) Removal of laminar bone superior to the foramen and the L4 accessory process reveals the distal dorsal root ganglion, recognized by its broader diameter.

Abbreviations: red arrows, dorsal root ganglion ( $L 5$ and L6); sap, superior articular processes; sp, spinous processes; tp, transverse processes; lam, laminar bone.

It is now clear that immune and glial cell responses alter neuronal function in the peripheral and central nervous system. ${ }^{17}$ Neurons in the DRG are surrounded by an envelope of satellite glial cells (SGCs), which carry receptors for numerous neuroactive agents and can therefore receive signals from other cells and respond to changes in their environment (Figure 3). Activation of SGCs might in turn influence neighboring neurons, and thus SGCs may participate in signal processing and transmission in sensory ganglia. Damage to the axons of sensory ganglia contributes to neuropathic pain. Such damage also affects SGCs so these cells have a role in the pathological changes in the ganglia. ${ }^{18}$

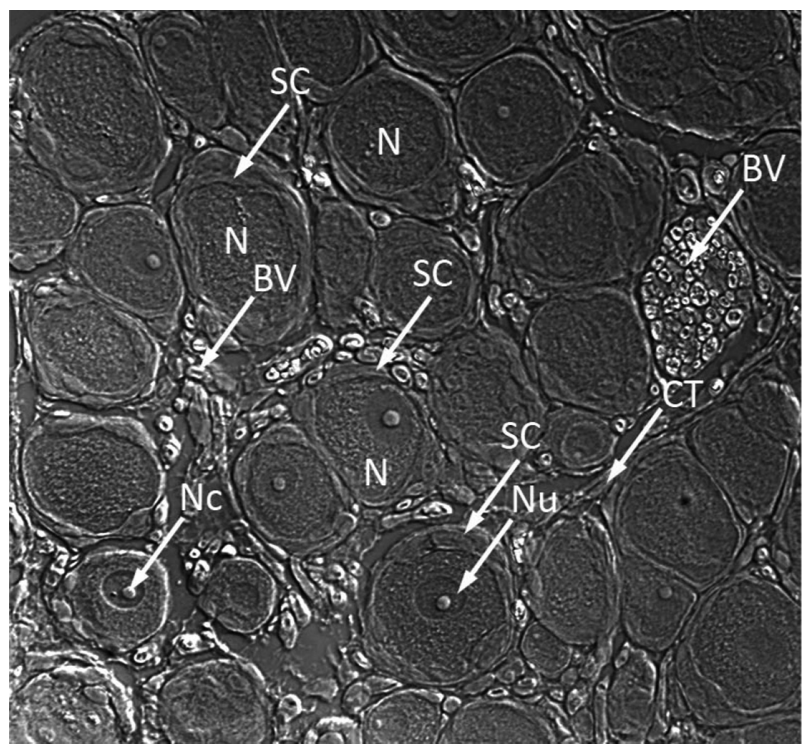

Figure 3 Microphotograph of dorsal root ganglion obtained from frozen section showing juxtaposition of DRG neurons and satellite cells.

Abbreviations: SC, satellite cells; $\mathrm{N}$, neurons; Nu, nucleolus; BV, blood vessels; $\mathrm{NC}$, nucleus; CT, connective tissue.

A major determinant of neuronal excitability is the extracellular concentration of potassium, which is regulated primarily by ion channels expressed by the SGCs that surround primary sensory neurons. ${ }^{18}$ Indeed, it has been shown that the impairment of glial potassium homeostasis in the sensory ganglia contributes to pain patomechanisms. ${ }^{19}$

After peripheral axotomy, there is an increased expression of neurotrophic factors such as nerve growth factor (NGF) and neurotrophin-3 (NT3) in satellite cells surrounding sensory neuron cell bodies in the DRG. These neurotrophins within the DRG trigger a persistent mechanical allodynia, showing that ganglia-derived neurotrophins are a source of nociceptive stimuli for neuropathic pain after peripheral nerve injury. ${ }^{20}$ This change may be sufficient to trigger the sprouting of sympathetic fibers within the DRG after peripheral nerve injury and in this way contribute to neuropathic pain. ${ }^{21}$

In a 2011 report, $\mathrm{Li}$ et al have shown that a single injection of corticosteroid in the vicinity of axotomized DRG can mimic many effects of systemic corticosteroid administration, mitigating behavioral and cellular abnormalities induced by spinal nerve ligation. Specifically, this treatment attenuated mechanical sensitivity, reduced sympathetic sprouting in the DRG, and decreased satellite glia activation in the DRG and microglia activation in the spinal cord after SNL. These findings provide further support for the idea that localized inflammation at the level of the DRG is an important component in neuropathic pain development. ${ }^{22}$ 


\section{DRG delivery in studies of pain mechanisms}

Different agents have been applied into the DRG in studies investigating pain mechanisms. In 1991, Lu and Richardson published a study in which an inflammatory reaction was provoked in rat DRG through the injection of Corynebacterium parvum and isogenous macrophages into the ganglion. The study concluded that changes favorable to axonal regeneration can be induced by products of inflammatory cells acting in the vicinity of the nerve cell body, and gave an indirect support to the idea that non-neuronal cells in the DRG might influence regenerative responses of primary sensory neurons. ${ }^{23}$

In an experimental model for chronic compression of the DRG a Ca ${ }^{2+}$ chelator and $\mathrm{K}^{+}$channel blocker were topically applied to the DRG to determine whether the effects on neuronal response differ for normal and chronically injured DRG neurons. ${ }^{12}$

DRG injection of isolectin B4 from Griffonia simplicifolia I (B4) conjugated to horseradish peroxidase (HRP) revealed that B4-HRP should be a suitable anterograde tracer of unmyelinated cutaneous and splanchnic but not muscle primary afferent fibers. ${ }^{24}$

Exposing rat DRG neurons to dibutyryl cAMP (db-cAMP) enables central branches to regenerate in the spinal cord by nullifying the ability of central nervous system myelin to inhibit elongation. It has been demonstrated that the intraganglionic injection of db-cAMP increases the expression of growth-associated tubulin isotypes, thus contributing to changes that may be necessary in order to increase intrinsic axon growth capacity. ${ }^{25}$ Further studies with intra-DRG injection of db-cAMP suggested that the beneficial actions of increased cyclic AMP activity on axonal regeneration of primary sensory neurons are mediated, at least in part, by the induction of neuropoietic cytokine synthesis within the dorsal root ganglion. ${ }^{26}$

DRG injection of a potent and selective TRPV1 receptor antagonist has helped in explaining how noxious thermal stimulation triggers the transmission of TRPV1-related signals to spinal wide dynamic range neurons in rats with peripheral inflammation. ${ }^{27}$

Our study of the role of neuropeptide Y (NPY) in pain modulation included the injection of NPY and its specific Y1 and Y2 receptor antagonists directly into the DRG. The exacerbation of pain-related behavior following NPY injection was accompanied by astrocyte activation in ipsilateral dorsal horn and with satellite cells activation in the DRG proximal to injury. This activation was reduced following Y2 receptor antagonist application. These findings indicate an important link between pain-related behavior and neuroimmune activation by NPY through its Y2 receptor. ${ }^{28}$

\section{Targeting DRG for studying existing and novel therapies}

To investigate the neurologic mechanisms of acidic local anesthetic-induced lower back pain in humans, Zhang et al administered bupivacaine and buffered saline at acidic or alkalinized $\mathrm{pH}$ at the L5 DRG of rats. The results demonstrated that acidic bupivacaine deposited at the DRG causes pain and hyperalgesia following the dissipation of local anesthetic effects. These findings may explain the limited therapeutic effects of some acidic local anesthetics used for the management of cancer-related and chronic back pain. ${ }^{29}$

A study of the selective migration and engraftment of bone marrow mesenchymal stem cells (MSCs) injected in rat lumbar DRG after sciatic nerve constriction showed the possible use of MSCs as a new therapeutic strategy for the treatment of peripheral nerve neuropathies. ${ }^{30}$

In the past decade we have witnessed the development of gene therapy for pain in the DRG. The targeted expression of foreign genes to the peripheral nerve system is interesting for many applications, including gene therapy of neuromuscular diseases, neuroanatomical studies, and elucidation of mechanisms of axonal flow. In 2000, Glatzel et al described a microneurosurgical technique for the injection of viral vectors into DRG. Vectors with transcriptional competence for DRG neurons led to the expression of the gene of interest. This study showed that DRG injection was vastly superior to the intraneural injection into the sciatic nerve. ${ }^{31}$

Fischer et al showed that the use of the DRG approach for the anatomically selective administration of a viral vector is associated with genetic modification of sensory neurons in the segment of the injected DRG, thus confirming the utility of the technique for research on sensory mechanisms and the possibility of therapeutic translation for the treatment of chronic pain. ${ }^{32}$ Yu et al showed the potential of lentivectors as a viable system for delivering target genes into DRGs to explore basic mechanisms of neuropathic pain, with the potential for future clinical use in the treatment of chronic pain. ${ }^{33}$

An interesting alternative to direct DRG gene therapy has recently been described. In 2008, Liu et al reported that subcutaneous inoculation of a novel human foamy virus (HFV) vector may be used to transfer the glutamic acid decarboxylase (GAD) gene to DRG cells to attenuate belowinjury level central neuropathic pain after spinal cord injury. ${ }^{34}$ Terashima et al genetically modified an adenovirus (Ad) to 
generate a helper virus (HV) that was detargeted for native adenoviral tropism and contained DRG homing peptides in the adenoviral capsid fiber protein. They used this HV to generate DRG-targeted helper-dependent Ad (HDAd). In mice, intrathecal injection of this HDAd produced a 100fold higher transduction of DRG neurons and a markedly attenuated inflammatory response compared with unmodified HDAd. This therapy showed promising results in an animal model of neuronopathy. ${ }^{35}$

\section{Experimental models for DRG injection}

However, targeted DRG delivery in small animal subjects is a methodological challenge, as DRGs are enclosed within the intervertebral foramen and are therefore not easily accessible for intraganglionic injection (Figure 2). ${ }^{36}$

Our group compared three methods of targeted DRG delivery, including percutaneous injection, intraganglionic injection after soft tissue removal and advancement of the needle into intervertebral foramen, and intraganglionic injection after laminar bone removal and exposure of the caudal pole of the DRG. ${ }^{36}$ Our results showed that intraganglionic injection after partial laminectomy resulted in a consistently successful injection into the DRG, as validated both with Coomassie blue and Fast Blue dyes. The removal of soft tissue but no bone, with blind insertion of the needle through the intervertebral foramen, is more challenging and less successful. Although it may take longer to achieve consistently successful results, the less traumatic approach of leaving the bone intact may have an advantage in creating less trauma and inflammation (Figure 2). ${ }^{36}$

Our analysis of the literature revealed that substantial methodological diversity characterizes published reports of DRG injection, probably due to difficulties faced by investigators when trying to access this structure. We also found that very few studies mention validation of DRG injections. ${ }^{36}$

In 2011, a technique for selective administration of an injected agent to a single DRG was further elaborated by Fischer et al. These findings indicate that direct injection of $2 \mu \mathrm{L}$ fills the adult rat DRG with only minor spread of the injectate to adjacent structures, produces minimal evidence of inflammatory changes, and has only mild and transient effects on sensory and motor function. ${ }^{32}$

The multitude of alternative techniques for intraganglionic injection has probably evolved to achieve specific experimental needs, but it may also be an indicator of a general inadequacy of the various methods. Our observation of the variability in results using approaches in which the
DRG is not exposed indicates that anatomic validation should be an obligatory component of research reports describing experiments using DRG injection. ${ }^{36}$

\section{Is DRG injection harmful?}

Whereas the histopathological and behavioral changes following peripheral nerve injury due to direct injection have been well investigated, little is known about the consequences of injection within the DRG or the adjacent spinal nerve. Potential clinical application requires a safe procedure that avoids inflammation or pain-related behavior.

Our group evaluated the neuroinflammatory response following a lidocaine or saline injection into the DRG or the adjacent spinal nerve. ${ }^{37}$ We hypothesized that an inflammatory response may follow local anesthetic injection into the DRG and produce hyperalgesia. In the experimental setting it has been reported that topical application of local anesthetic to the DRG induces mechanical hyperalgesia in the rat, ${ }^{29}$ but possible morphological changes in the DRG have not been studied. We have found that the injection of lidocaine into a DRG induced pain-related behavior and a marked inflammatory response in the DRG. ${ }^{37}$ It is important that we also identified an inflammatory response in saline-injected rats, but this was not accompanied with marked hyperalgesia, perhaps because this inflammatory response was significantly lower than after the lidocaine injection suggesting that DRG injection can be a secure procedure. ${ }^{37}$

Our findings raise the possibility that hyperalgesia after injection into the DRG may be attributed to an inflammatory response. ${ }^{37}$ The inflammation was evaluated through the activation of satellite and glial cells. The activation of these cells was evaluated through the expression of glial fibrillary acidic protein (GFAP). The activation of satellite cells is not specific for neuroinflammation, however, the lack of other possible reasons for its activation can help us attribute this activation to inflammatory processes. The activation was exhibited as rings of immunopositive satellite cells around neurons. Other markers of inflammation, like OX-42 immunopositive cells, which represent activated microglia, or Pan-T positive cells, which indicate activated $\mathrm{T}$ lymphocytes, were not observed in saline injected rats. ${ }^{37}$ These findings indicate that lidocaine injection into the DRG activates resident immunological cells, but it does not produce significant immigration of nonresident inflammatory cells into the DRG.

In addition to the inflammatory response, it is important to validate the behavioral consequences of DRG injection and to test behavioral responses following these procedures. We have demonstrated already that targeted drug delivery 
by direct intraganglionar injection per se does not produce behavioral changes in rats. ${ }^{36}$

Recently we have also shown that small differences in surgical approach can result in significant behavioral differences. ${ }^{38}$ Specifically, we investigated whether there is a direct relationship between the extent of laminectomy and the development of mechanical hypersensitivity after ganglionectomy. We found hyperalgesia in rats with extensive laminectomy, regardless of performance or absence of ganglionectomy, while in rats with minimal laminectomy there was no increase in pain-related behavior. ${ }^{38}$

Based on these results it could be inferred that partial laminectomy is a rather precise method of injecting DRG and does not produce measurable behavioral differences. The observed variability in results using approaches in which the DRG was not exposed indicates that anatomic validation should be a part of the experimental procedure using DRG injection.

\section{Limitations in ganglion approach}

A limitation for DRG delivery is the location of the ganglion, which makes it difficult to reach. DRGs are protected by the bony structures of the vertebra. To reach DRGs in animal models, one needs to drill a hole in the bone covering the ganglion. In humans, due to larger structures, it is possible to access most of the DRGs via intervertebral foramen by carefully positioning a patient and navigating a needle using radiographic or ultrasound imaging, as shown by several approaches already used in a clinical setting. Pulsed radiofrequency (RF) of DRGs is a therapeutic procedure during which a heat lesion is placed sufficiently near the DRG to produce what is called a partial lesion, but not so close as to completely destroy the ganglion. ${ }^{39}$ Selective nerve root blocks (SNRBs) have been used for many years as a diagnostic tool in patients with lower back pain with radicular symptoms, as well as for pain relief. This technique also involves structures beyond the intervertebral foramen. However, patient responses to SNRBs are often non specific and pain relief after injecting local anesthetic is often difficult to interpret. ${ }^{40}$

The DRG can be injured during SNRBs. ${ }^{9}$ However, these injections into the DRG are well tolerated. The cervical level is a very important exception since there is a very short length from the DRG to the spinal cord, where arrival of the injected drug may produce disastrous cord damage. ${ }^{11}$

\section{Alternatives to DRG targeted delivery of drugs}

The idea to target specific parts of the nervous system in order to deliver therapeutics is not new, as shown by the principles of regional anesthesia. Within anesthetic practice, the role of regional anesthesia - including peripheral nerve block has expanded greatly over the past two decades. In 1998, a national survey demonstrated that $88 \%$ of US anesthesiologists make use of regional techniques. ${ }^{41}$

Regional nerve blockade, or more commonly nerve block, is a general term used to refer to the injection of a local anesthetic onto or near the nerves for temporary control of pain. The idea of Johannes P Mueller (1801-1858) that nerves determine what the mind perceives sparked interest in nerve function. ${ }^{42}$ By 1845, Sir Frances Rynd (1801-1861) had already delivered a morphine solution to a nerve in an effort to relieve intractable neuralgia, which appears to be the first documented nerve block as it is defined today. ${ }^{43}$ However, Rynd used a cannula to deliver a solution by means of gravity. Ten years later, in 1855, Alexander Wood (1817-1884) used a graduated glass syringe and needle to perform the same procedure. ${ }^{44}$

Single-shot peripheral nerve blocks are mostly used for a variety of surgical procedures. A continuous peripheral nerve block, also termed "perineural local anesthetic infusion," involves the percutaneous insertion of a catheter adjacent to a peripheral nerve, followed by local anesthetic administration via the catheter, providing anesthesia for multiple days or even months. ${ }^{45}$

Complications of peripheral nerve blocks include easily rectified minor complications that occur frequently, but major risks include nerve injury, catheter infection, bleeding, and local anesthetic systemic toxicity (LAST). ${ }^{46}$

Steroids may be given with or without anesthetics during peripheral nerve blocks. The clinical introduction of cortisone in 1949 revolutionized medical care for patients with various diseases. The first neuroaxial application of steroids in epidural injections was already described in 1952. A variety of corticosteroid agents have been applied neuroaxially to treat spinal pain and other types of painful conditions, since it has been postulated that corticosteroids reduce inflammation by inhibiting either the synthesis or the release of a number of pro-inflammatory substances as well as by causing a reversible local anesthetic effect. ${ }^{47}$

The perineural approach has also been described for other drugs, such as the introduction of the local injection perineural therapy of ipidacrinum for focal neuropathies of upper extremities, which exhibited significantly better results compared to intramuscular injections of the same drug. ${ }^{48}$

However, it should be noted that ectopic nerve activity occurs at the dorsal root ganglion and that is why peripheral nerve block may fail and perineural drug infusion may be ineffective for neuropathic pain treatment. 
Sympathetic block is also a well-known treatment in clinical practice. However, although there is a widely held view that sympathetic nerve blocks reduce incidence of pain in acute herpes zoster and postherpetic neuralgia, a critical review of the literature found little support for this notion. ${ }^{49}$

Epidural pathway is another option for targeted drug delivery. Epidural steroid injections have been used for more than 50 years to treat lower back pain and they are the most common intervention in pain clinics throughout the world. ${ }^{50}$

About a third of chronic lower back pain patients have predominantly neuropathic pain. ${ }^{51}$ However, in 2011, Iversen and colleagues reported the outcomes of a multicenter randomized controlled trial, in which they concluded that caudal epidural steroids are not recommended for chronic lumbar radiculopathy. ${ }^{52}$

Intrathecal drug delivery is another interventional mode of pain treatment. However, most reported benefits of systemic and regional analgesics and interventional approaches to pain relief are not based on randomized trials and are subject to selection bias, sampling error, and placebo responses, which may over-inflate reported benefits. Randomized controlled trials are needed to confirm reported benefits. ${ }^{53}$ While intrathecal injection is a simple and relatively noninvasive means to deliver agents to sensory neurons, it is not possible to restrict the injected solution to a single side or to a limited longitudinal range of vertebral levels. ${ }^{32}$

Although multiple types of targeted interventional therapies have been used in practice, needle trauma associated with these treatments may cause neuropathic pain itself. ${ }^{54,55}$ Therefore, exploring new options for interventional pain management of neuropathic pain could be potentially useful for clinical practice.

\section{Conclusion}

The animal model for DRG targeted delivery of drugs is a valuable tool in pain research. Such an approach may provide adequate specificity to capitalize on the new knowledge of peripheral sensory nerve function in painful conditions. The development of new techniques for sustained infusions at the level of a single DRG and gene delivery using viral vectors are probable new venues for the regional therapeutic approach at the level of a single DRG.

\section{Acknowledgments}

All reviewed work was supported by the Ministry of Science, Education, and Sports of the Republic of Croatia grant no. 216-2160528-0522 and National Foundation for Science,
Higher Education and Technological Development of the Republic of Croatia grant no. 02.05./28.

\section{Disclosure}

The authors have no financial or other relationship to report that might lead to a conflict of interest.

\section{References}

1. Brennan F, Carr DB, Cousins M. Pain management: a fundamental human right. Anesth Analg. 2007;105(1):205-221.

2. Finnerup NB, Otto M, McQuay HJ, Jensen TS, Sindrup SH. Algorithm for neuropathic pain treatment: an evidence based proposal. Pain. 2005;118(3):289-305.

3. Sapunar D, Puljak L. What can rats tell us about neuropathic pain? Critical evaluation of behavioral tests used in rodent pain models. Periodicum Biologorum. 2009;111(2):155-160.

4. Mogil JS. Animal models of pain: progress and challenges. Nat Rev Neurosci. 2009;10(4):283-294.

5. Sapunar D, Ljubkovic M, Lirk P, McCallum JB, Hogan QH. Distinct membrane effects of spinal nerve ligation on injured and adjacent dorsal root ganglion neurons in rats. Anesthesiology. 2005;103(2):360-376.

6. Xie WR, Deng H, Li H, Bowen TL, Strong JA, Zhang JM. Robust increase of cutaneous sensitivity, cytokine production and sympathetic sprouting in rats with localized inflammatory irritation of the spinal ganglia. Neuroscience. 2006;142(3):809-822.

7. Abram SE, Yi J, Fuchs A, Hogan QH. Permeability of injured and intact peripheral nerves and dorsal root ganglia. Anesthesiology. 2006; 105(1):146-153.

8. Jimenez-Andrade JM, Herrera MB, Ghilardi JR, Vardanyan M, Melemedjian OK, Mantyh PW. Vascularization of the dorsal root ganglia and peripheral nerve of the mouse: implications for chemicalinduced peripheral sensory neuropathies. Mol Pain. 2008;4:10.

9. Pfirrmann CW, Oberholzer PA, Zanetti M, et al. Selective nerve root blocks for the treatment of sciatica: evaluation of injection site and effectiveness - a study with patients and cadavers. Radiology. 2001; 221(3):704-711.

10. Peters CM, Jimenez-Andrade JM, Jonas BM, et al. Intravenous paclitaxel administration in the rat induces a peripheral sensory neuropathy characterized by macrophage infiltration and injury to sensory neurons and their supporting cells. Exp Neurol. 2007;203(1):42-54.

11. Hogan QH. Labat lecture: the primary sensory neuron: where it is, what it does, and why it matters. Reg Anesth Pain Med. 2010;35(3): 306-311.

12. Hu SJ, Xing JL. An experimental model for chronic compression of dorsal root ganglion produced by intervertebral foramen stenosis in the rat. Pain. 1998;77(1):15-23.

13. DeLeo JA, Winkelstein BA. Physiology of chronic spinal pain syndromes: from animal models to biomechanics. Spine. 2002;27(22): 2526-2537.

14. Znaor L, Lovric S, Hogan Q, Sapunar D. Association of neural inflammation with hyperalgesia following spinal nerve ligation. Croat Med J. 2007;48(1):35-42.

15. Ueda H. Molecular mechanisms of neuropathic pain-phenotypic switch and initiation mechanisms. Pharmacol Ther. 2006;109(1-2):57-77.

16. Wang W, Gu J, Li YQ, Tao YX. Are voltage-gated sodium channels on the dorsal root ganglion involved in the development of neuropathic pain? Mol Pain. 2011;7:16.

17. Scholz J, Woolf CJ. The neuropathic pain triad: neurons, immune cells and glia. Nat Neurosci. 2007;10(11):1361-1368.

18. Hanani M. Satellite glial cells in sensory ganglia: from form to function. Brain Res Brain Res Rev. 2005;48(3):457-476.

19. Takeda M, Takahashi M, Nasu M, Matsumoto S. Peripheral inflammation suppresses inward rectifying potassium currents of satellite glial cells in the trigeminal ganglia. Pain. 2011;152(9):2147-2156. 
20. Zhou XF, Deng YS, Xian CJ, Zhong JH. Neurotrophins from dorsal root ganglia trigger allodynia after spinal nerve injury in rats. Euro J Neurosci. 2000;12(1):100-105.

21. Ramer MS, Thompson SW, McMahon SB. Causes and consequences of sympathetic basket formation in dorsal root ganglia. Pain. 1999; Suppl 6:S111-S120.

22. Li JY, Xie W, Strong JA, Guo QL, Zhang JM. Mechanical hypersensitivity, sympathetic sprouting, and glial activation are attenuated by local injection of corticosteroid near the lumbar ganglion in a rat model of neuropathic pain. Reg Anesth Pain Med. 2011;36(1):56-62.

23. Lu X, Richardson PM. Inflammation near the nerve cell body enhances axonal regeneration. J Neurosci. 1991;11(4):972-978.

24. Wang HF, Robertson B, Grant G. Anterograde transport of horseradishperoxidase conjugated isolectin B4 from Griffonia simplicifolia I in spinal primary sensory neurons of the rat. Brain Res. 1998;811(1-2): 34-39.

25. Han PJ, Shukla S, Subramanian PS, Hoffman PN. Cyclic AMP elevates tubulin expression without increasing intrinsic axon growth capacity. Exp Neurol. 2004;189(2):293-302.

26. Wu D, Zhang Y, Bo X, et al. Actions of neuropoietic cytokines and cyclic AMP in regenerative conditioning of rat primary sensory neurons. Exp Neurol. 2007;204(1):66-76.

27. McGaraughty S, Chu KL, Faltynek CR, Jarvis MF. Systemic and sitespecific effects of A-425619, a selective TRPV1 receptor antagonist, on wide dynamic range neurons in CFA-treated and uninjured rats. J Neurophysiol. 2006;95(1):18-25.

28. Sapunar D, Vukojevic K, Kostic S, Puljak L. Attenuation of pain-related behavior evoked by injury through blockade of neuropeptide Y Y2 receptor. Pain. 2011;152(5):1173-1181.

29. Zhang JM, Homma Y, Ackerman WE, Brull SJ. Topical application of acidic bupivacaine to the lumbar ganglion induces mechanical hyperalgesia in the rat. Anesth Analg. 2001;93(2):466-471.

30. Coronel MF, Musolino PL, Villar MJ. Selective migration and engraftment of bone marrow mesenchymal stem cells in rat lumbar dorsal root ganglia after sciatic nerve constriction. Neurosci Lett. 2006;405(1-2): 5-9.

31. Glatzel M, Flechsig E, Navarro B, et al. Adenoviral and adeno-associated viral transfer of genes to the peripheral nervous system. Proc Natl Acad Sci US A. 2000;97(1):442-447.

32. Fischer G, Kostic S, Nakai H, et al. Direct injection into the dorsal root ganglion: technical, behavioral, and histological observations. J Neurosci Methods. 2011;199(1):43-55.

33. Yu H, Fischer G, Jia G, Reiser J, Park F, Hogan QH. Lentiviral gene transfer into the dorsal root ganglion of adult rats. Mol Pain. 2011;7:63.

34. Liu W, Liu Z, Liu L, et al. A novel human foamy virus mediated gene transfer of GAD67 reduces neuropathic pain following spinal cord injury. Neurosci Lett. 2008;432(1):13-18.

35. Terashima T, Oka K, Kritz AB, Kojima H, Baker AH, Chan L. DRGtargeted helper-dependent adenoviruses mediate selective gene delivery for therapeutic rescue of sensory neuronopathies in mice. J Clin Invest. 2009;119(7):2100-2112.
36. Puljak L, Kojundzic SL, Hogan QH, Sapunar D. Targeted delivery of pharmacological agents into rat dorsal root ganglion. $J$ Neurosci Methods. 2009;177(2):397-402.

37. Puljak L, Kojundzic SL, Hogan QH, Sapunar D. Lidocaine injection into the rat dorsal root ganglion causes neuroinflammation. Anesth Analg. 2009;108(3):1021-1026.

38. Kosta V, Kojundzic SL, Sapunar LC, Sapunar D. The extent of laminectomy affects pain-related behavior in a rat model of neuropathic pain. Eur J Pain. 2009;13(3):243-248.

39. Van Kleef M, Spaans F, Dingemans W, Barendse GA, Floor E, Sluijter ME. Effects and side effects of a percutaneous thermal lesion of the dorsal root ganglion in patients with cervical pain syndrome. Pain. 1993;52(1):49-53.

40. Haynsworth RF Jr. Selective nerve root blocks: a new technique using electrical stimulation. Pain Physician. 2003;6(4):517-520.

41. Hadzic A, Vloka JD, Kuroda MM, Koorn R, Birnbach DJ. The practice of peripheral nerve blocks in the United States: a national survey [p2e comments]. Reg Anesth Pain Med. 1998;23(3):241-246.

42. Riese W, Arrington GE Jr. The history of Johannes Mueller's doctrin of the specific energies of the senses: original and later versions. Bull Hist Med. 1963;37:179-183.

43. Rynd F. Neuralgia - introduction of fluid to the nerve. Dublin Med Press. 1845;13:167-168.

44. Wood A. New method of treating neuralgia by the direct application of opiates to the painful points. Edinb Med Surg J. 1855;82:265-281.

45. Ilfeld BM. Continuous peripheral nerve blocks in the hospital and at home. Anesthesiol Clin. 2011;29(2):193-211.

46. Jeng CL, Torrillo TM, Rosenblatt MA. Complications of peripheral nerve blocks. Br J Anaesth. 2010;Suppl 1:i97-i107.

47. Manchikanti L. Role of neuraxial steroids in interventional pain management. Pain Physician. 2002;5(2):182-199.

48. Shirokov VA, Bakhtereva EV, Leiderman EL. Focal neuropathies: new possibilities for pharmacological treatment. Zh Nevrol Psikhiatr Im S S Korsakova. 2011;111(6):49-52. Russian.

49. Boas RA. Sympathetic nerve blocks: in search of a role. Reg Anesth Pain Med. 1998;23(3):292-305

50. Manchikanti L. The growth of interventional pain management in the new millennium: a critical analysis of utilization in the medicare population. Pain Physician. 2004;7(4):465-482.

51. Freynhagen R, Baron R, Gockel U, Tolle TR. painDETECT: a new screening questionnaire to identify neuropathic components in patients with back pain. Curr Med Res Opin. 2006;22(10):1911-1920.

52. Iversen T, Solberg TK, Romner B, et al. Effect of caudal epidural steroid or saline injection in chronic lumbar radiculopathy: multicentre, blinded, randomised controlled trial. BMJ. 2011;343:d5278.

53. Davis MP. Recent advances in the treatment of pain. F1000 Med Rep. 2010;2:63.

54. Ramos GC, Gomes EC. Neuropathic pain after epidural needle trauma. Rev Bras Anestesiol. 2008;58(4):380-386.

55. Hashimoto A, Ito H, Harato M, Fujiwara Y, Komatsu T. Complications of peripheral nerve block. Masui. 2011;60(1):111-119. Japanese.

Journal of Pain Research

\section{Publish your work in this journal}

The Journal of Pain Research is an international, peer-reviewed, open access, online journal that welcomes laboratory and clinical findings in the fields of pain research and the prevention and management of pain. Original research, reviews, symposium reports, hypothesis formation and commentaries are all considered for publication.

The manuscript management system is completely online and includes a very quick and fair peer-review system, which is all easy to use. Visit http://www.dovepress.com/testimonials.php to read real quotes from published authors.

\section{Dovepress}

Submit your manuscript here: http://www.dovepress.com/journal-of-pain-research-journal 